\title{
Spectrometer for cluster ion beam induced luminescence.
}

\section{$\operatorname{AUTHOR}(\mathrm{S})$ :}

Ryuto, H; Musumeci, F; Sakata, A; Takeuchi, M; Takaoka, G H

\section{CITATION:}

Ryuto, H ... [et al]. Spectrometer for cluster ion beam induced luminescence.. Review of scientific instruments 2015, 86(2): 023106.

\section{ISSUE DATE:}

2015-02-11

URL:

http://hdl.handle.net/2433/196925

\section{RIGHT:}

(c) 2015 American Institute of Physics. This article may be downloaded for personal use only. Any other use requires prior permission of the author and the American Institute of Physics. The following article has been submitted to/accepted by Review of scientific instruments. After it is published, it will be found at http://scitation.aip.org/content/aip/journal/rsi 


\title{
Spectrometer for cluster ion beam induced luminescence
}

\author{
H. Ryuto, ${ }^{1, a), b)}$ F. Musumeci, ${ }^{2,3}$ A. Sakata, ${ }^{1}$ M. Takeuchi, ${ }^{1}$ and G. H. Takaoka ${ }^{1}$ \\ ${ }^{1}$ Photonics and Electronics Science and Engineering Center, Kyoto University, Kyoto 615-8510, Japan \\ ${ }^{2}$ Department of Physics and Astronomy, Catania University, Catania 95123, Italy \\ ${ }^{3}$ INFN Laboratori Nazionali del Sud, Catania 95123, Italy
}

(Received 26 November 2014; accepted 23 January 2015; published online 11 February 2015)

\begin{abstract}
A spectrometer to detect the ultra-weak luminescence originated by the collision of cluster ions on the surfaces of solid materials was constructed. This spectrometer consists of 11 photomultipliers with band-pass interference filters that can detect the luminescence within the wavelength ranging from 300 to $700 \mathrm{~nm}$ and of a photomultiplier without filter. The calibration of the detection system was performed using the photons emitted from a strontium aluminate fluorescent tape and from a high temperature tungsten filament. Preliminary measurements show the ability of this spectrometer to detect the cluster ion beam induced luminescence. (C) 2015 AIP Publishing LLC. [http://dx.doi.org/10.1063/1.4907540]
\end{abstract}

\section{INTRODUCTION}

Ion beams are widely employed in the semiconductor industry. Some, such as boron, phosphorus, and arsenic ion beams, are used for ion implantation while some others, such as argon and gallium ion beams, are used for processing. The ion beams are also utilized as a probe in the surface analysis. The typical example is the Rutherford backscattering spectrometry, which generally uses proton or helium ion beam at a few MeV. Another technique for detecting surface property has been studied. ${ }^{1,2}$ It utilizes the luminescence induced by irradiation of ion beams at $\mathrm{MeV}$ energy range. The ion beam induced luminescence depends on the impurities and imperfections within the near surface region of various solid materials. ${ }^{1,3}$

In addition to the properties of these conventional atomic ion beams, the irradiation effects of cluster ion beams have been studied. ${ }^{4,5}$ The clusters, which are the aggregates of atoms or molecules bound by intermolecular forces, usually have extremely large mass-to-charge ratio. This allows to apply ions much slower than that obtained with atomic ion beams, produced with the same extraction voltage. Because of this low speed irradiation effect, the lattice disorder, induced by the irradiation of cluster ion beams accelerated with several $\mathrm{kV}$ voltages, is small even when the sputtering yield is a few orders of magnitude larger than that of the atomic ion beams. ${ }^{5}$ The irradiation effects of cluster ions are also characterized by the high density collisions of atoms or molecules against the target. According to the molecular dynamics calculations, due to this high density collision, the effective temperature of the few tens nm diameter area, where a cluster collides, increases to a few thousand $\mathrm{K}^{6,7}$ Due to the temperature increase, the enhancement of the chemical interactions between the molecules that comprise the clusters and the target is expected. At the same acceleration voltage,

\footnotetext{
a) Author to whom correspondence should be addressed. Electronic mail: ryuto@kuee.kyoto-u.ac.jp

b) Formerly, H. Akiyoshi.
}

the kinetic energy per molecule of the clusters is several orders of magnitude smaller than that of the ions of the atomic ion beams, usually used for the detection of the ion beam induced luminescence. Nevertheless, the high temperatures foreseen by MD calculations suggest the possibility that some photonic emission could took place during the collision process. As a matter of fact, in a previous paper, we reported the detection of photons during the irradiation of silicon and gold targets by water cluster ions accelerated at $9 \mathrm{kV}{ }^{8}$ Our previous system does not permit to achieve spectral information, which would be very useful to better understand the origin of this phenomenon. On the other hand, no conventional spectrometer could be able to give us such information because the number of photons induced by the collision of the cluster ions, accelerated at several $\mathrm{kV}$, is too small. This forced us to develop a spectrometer, which makes use of 11 pairs of photomultipliers and optical filters.

\section{CLUSTER ION BEAM APPARATUS}

Water and argon cluster ion beams were used to examine the operation of the cluster ion beam luminescence apparatus. Both cluster ion beams were produced by the adiabatic expansion method..$^{9}$ In the previous studies, ${ }^{10,11}$ the apparatuses were selected from two kinds of apparatuses that have been optimized for the production of cluster ions produced from the source materials in gas and liquid phases. In this study, the apparatus for the liquid source materials was used to produce not only water cluster ions but also argon cluster ions to enable the comparison between the spectra induced by water and argon cluster ions using the same configuration of the detectors. Figure 1 shows a schematic view of the apparatus together with a close up view of the photon detectors. The apparatus consists of three vacuum chambers. In the case of water cluster, purified water was filled into the source material container, placed inside the first vacuum chamber, and heated to increase the vapor pressure using a heater attached on the outer wall of the container. The typical pressure inside the source was $0.30 \pm 0.09 \mathrm{MPa}$. 
It was monitored using a manometer placed outside of the vacuum chamber. In the case of argon cluster, argon gas was filled in the source material container typically at a pressure of $0.45 \pm 0.02 \mathrm{MPa}$. The water vapor or the argon gas was ejected to the vacuum chamber through a supersonic nozzle to produce clusters. The clusters were ionized by electron ionization at the second vacuum chamber. Another vacuum chamber placed between the first and second chambers was used for differential pumping. A skimmer was placed between this vacuum chamber and the first vacuum chamber to prevent the clusters from disintegration caused by shockwaves. A loop of oxide filament cathode was used for electron ionization. ${ }^{8}$ The monomers and small clusters were eliminated from the beam by the retarding voltage method. This method is based on the phenomenon that the velocity of the clusters produced by the adiabatic expansion method is uniform typically within $10 \% .^{9,12}$ The retarding voltages were 14 and $9 \mathrm{~V}$ for water and argon clusters, respectively. These retarding voltages correspond to the cluster size of 100 molecules both for water and argon clusters. The mode of the cluster size distributions both of water and argon cluster ion beams measured by the time of flight method was approximately $3 \times 10^{3}$ molecules when singly charged cluster ions were assumed. This assumption was made because it is reported that the electron ionization of weakly bound systems produces fragments rather than multiply charged ions. ${ }^{13,14}$

The typical acceleration voltage was from 1 to $9 \mathrm{kV}$. The transverse divergence of the beam was suppressed using an einzel lens. An antireflection material covered the inside wall of the einzel lens and the second vacuum chamber. Stainless steel sheets painted with Okitsumo B-600 paint were used as antireflection material. ${ }^{8}$ The paint was also used to decrease the reflectivity of the surface of the beam collimator described below. The accelerated cluster ion beam was irradiated on a sample attached on a linear motion feedthrough. A 20-mmdiameter collimator to reject the background light and an 8-mm-diameter beam collimator were placed between the einzel lens and sample. The current density of the cluster ion beam was measured using a Faraday cup, also attached at the linear motion feedthrough. A voltage of approximately $-300 \mathrm{~V}$ was applied for electron suppression.

\section{PHOTON DETECTION}

A photons detection system, consisting of 12 photomultipliers, together with band-pass optical filters, was placed at upstream of the sample. The photomultipliers were attached on one of the 2 photomultiplier holders placed at left (L) and right $(\mathrm{R})$ side of the beam axis in vertical position. The angle between the beam axis and photomultiplier holders was $45^{\circ}$, and the distance between the center of the photomultiplier holder and the center of the sample was $60 \mathrm{~mm}$. The photomultipliers are numbered clockwise from L1 (R1) at the top to L6 (R6) as shown in Fig. 1. Every photomultiplier holder contained six photomultipliers. ${ }^{15}$ Eleven band-pass filters, ${ }^{16}$ with central wavelengths from 340 to $667 \mathrm{~nm}$, were attached in front of the photomultipliers. To suppress the effect of infrared (IR) leakage of the band-pass filters, two types of photomultipliers, R7400P-01 with multialkali photocathode and R7400P with bialkali photocathode, were used in this detection system.

Table I shows the combinations of the photomultipliers and band-pass filters. The R7400P-01 photomultipliers have higher quantum efficiency in longer wavelength range than R7400P photomultipliers. Thus, the R7400P-01 photomultipliers were combined with the band-pass filters with longer central wavelengths. On the other hand, the R7400P photomultipliers were combined with the band-pass filters with shorter central wavelengths to avoid the IR leakage of the band-pass filters in longer wavelength ranges. Figure 2 shows the wavelength dependence of the total efficiency of the system. The total efficiencies were calculated by multiplying the

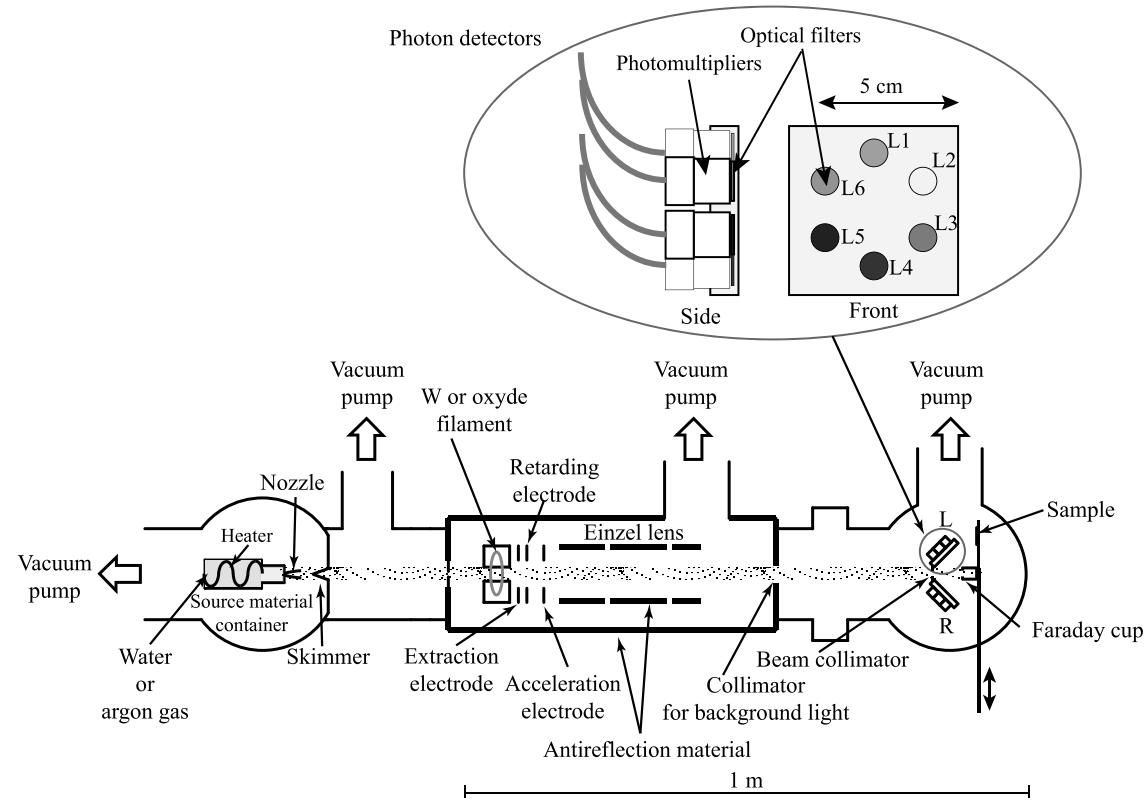

FIG. 1. Schematic view of spectrometer for cluster ion beam induced luminescence placed in cluster ion beam irradiation apparatus. 
TABLE I. Combinations of photomultiplier types and band-pass filters attached on left and right photomultiplier holders. The positions, L1, L2, L3, L4, L5, and L6, are indicated in Fig. 1. The positions, R1, R2, R3, R4, R5, and R6, are the corresponding position to these in the right photomultiplier holder.

\begin{tabular}{llc}
\hline \hline $\begin{array}{l}\text { Position of } \\
\text { photomultiplier }\end{array}$ & Type of photomultiplier & $\begin{array}{c}\text { Central wavelength of } \\
\text { optical filter (nm) }\end{array}$ \\
\hline L1 & R7400P & 377 \\
L2 & R7400P & 468 \\
L3 & R7400P & 510 \\
L4 & R7400P-01 & No filter \\
L5 & R7400P & 340 \\
L6 & R7400P & 487 \\
R1 & R7400P-01 & 568 \\
R2 & R7400P-01 & 623 \\
R3 & R7400P-01 & 533 \\
R4 & R7400P & 429 \\
R5 & R7400P-01 & 667 \\
R6 & R7400P-01 & 590 \\
\hline \hline
\end{tabular}

quantum efficiencies provided by the production company and the transmittance of the band-pass filters measured using Shimadzu MPS-2000 multi-purpose spectrometer. The signals from the photomultipliers were transformed into normalized logic signals by two EG\&G CF8000 discriminators and three NIM-TTL CAEN N89 converters and, then, counted by National Instruments PCI-6601 and PCI-6602 counters.

This spectrometric system exhibits a very high sensitivity compared with ordinary spectrometers. A typical CCD spectrometer has a maximum $200 \mu \mathrm{m}$ wide entrance aperture and a dark counts rate of about 5 counts/s. The sensitivity is approximately 50 photons/count. So, in order to have a signal of the order of background, it requires a flux density of about 8000 photons/( $\left.\mathrm{s} \mathrm{mm}^{2}\right)$. Each of our photomultipliers has a dark counts rate of approximately 10 counts/s, a sensitive area of about $70 \mathrm{~mm}^{2}$, and an average quantum efficiency

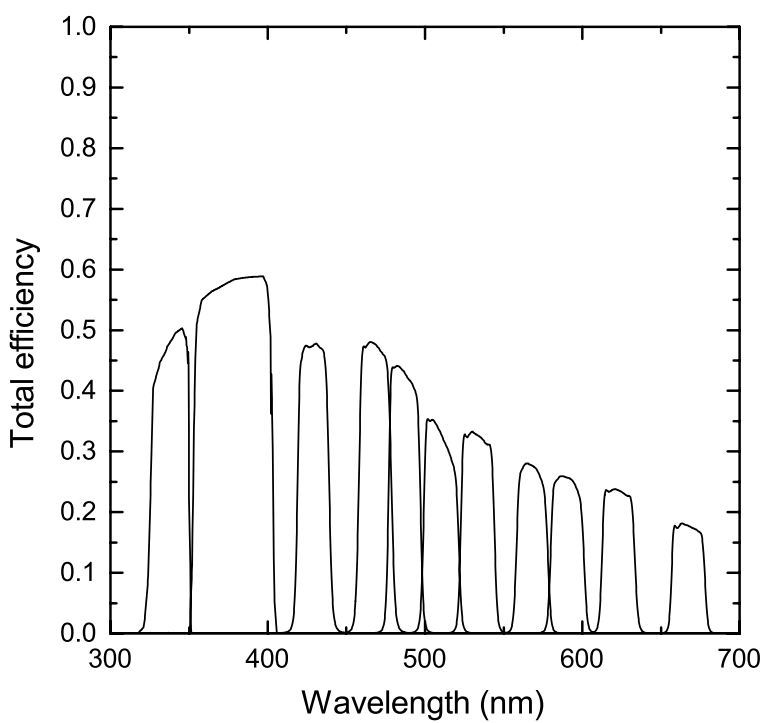

FIG. 2. Wavelength dependence of quantum efficiencies of photomultipliers multiplied by transmittance of optical filters. of about $5 \%$. Thus, in order to have a signal of the order of background, it requires a flux density of about 3 photons/(s $\mathrm{mm}^{2}$ ). Therefore, it is about 3000 times more sensitive than a CCD spectrometer. If one considers that we use 12 of these photomultipliers increasing the total area of the device, the sensitivity of the system would be approximately 36000 times higher than that of the CCD spectrometer.

\section{CALIBRATION OF DETECTION SYSTEM}

The differences in the photomultiplier types and solid angles covered by photomultipliers were normalized by comparing the counting rates measured with 12 photomultipliers. At first, a 9-mm-diameter strontium aluminate fluorescent tape $\mathrm{e}^{17}$ was adhered at the position of the sample. The fluorescent spectrum from the tape had a broad single peak centered on $520 \mathrm{~nm}$. The light from the fluorescent tape was measured with the photomultipliers without band-pass filters. A set of normalization factors was determined by assuming a Lambertian distribution. The difference in the sensitivities between R7400P and R7400P-01 photomultipliers, in the spectral range of the fluorescent spectrum, $32 \%$, was also included in the normalization factors. The fluorescent spectrum measured using a spectrometer (OceanOptics HR4000) and the quantum efficiency curves were used to estimate the sensitivity difference. This set of normalization factors allows us to estimate the angular distribution of cluster-ion-beam induced luminescence.

Further calibration of the detection system was performed by using the light emitted from a tungsten filament. The oxide filament for the ionization of clusters was replaced with a loop of tungsten wire, whose operating temperature was much higher than that of the oxide filament. The diameter of the tungsten wire was $0.35 \mathrm{~mm}$, and the diameter of the

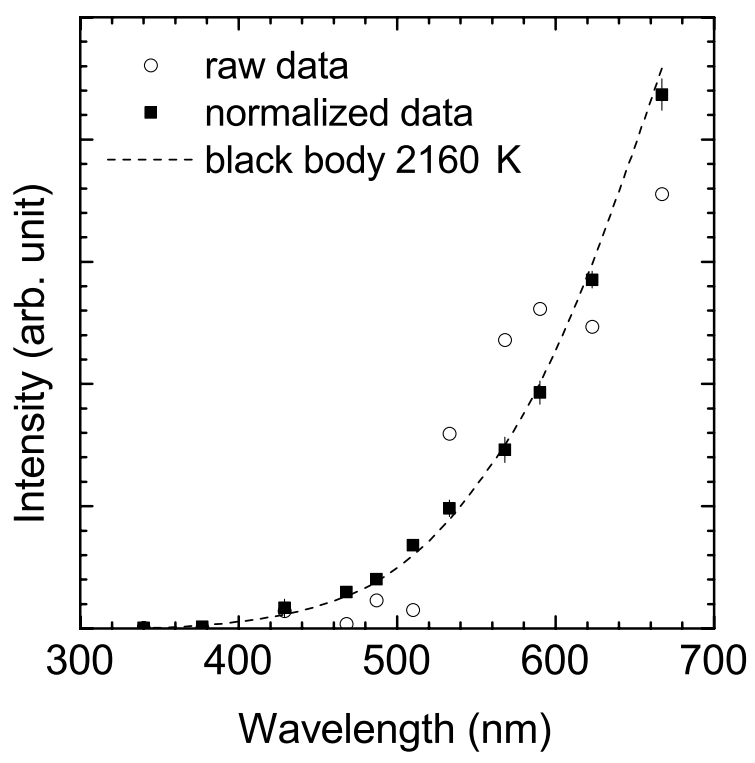

FIG. 3. Spectrum of photons from lower temperature tungsten filament measured with present detection system. The open circles, filled squares, and dashed line represent the data before normalization, normalized data, and black body spectrum at $2160 \mathrm{~K}$ that reproduced separately measured spectrum using HR4000 spectrometer, respectively. 


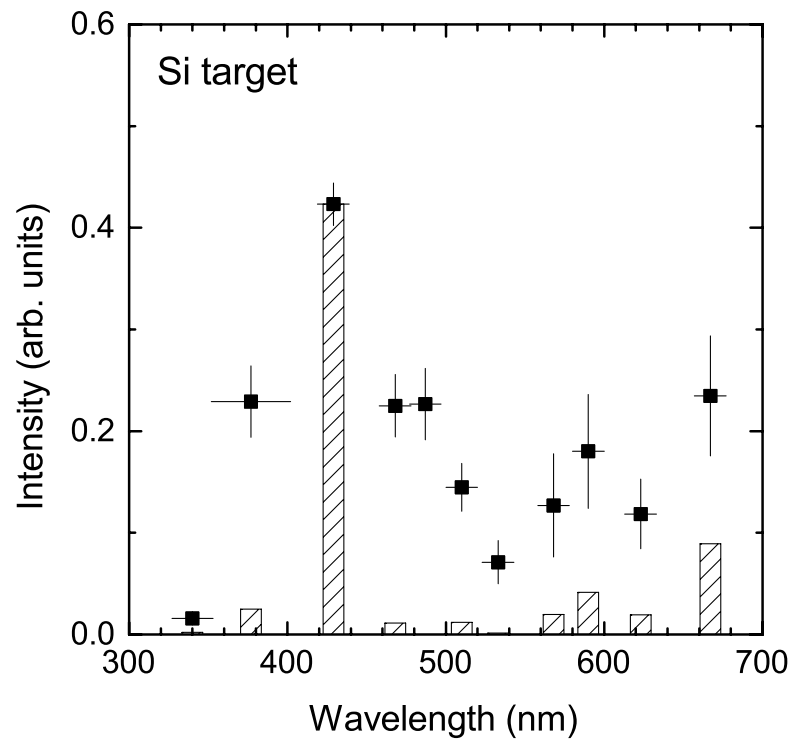

FIG. 4. Spectrum of photons, emitted during the collision of Ar cluster ion beam and Si surface, obtained using present detection system (square) together with Ar spectrum from NIST data tables.

filament loop was $50 \mathrm{~mm}$. The spectra from the filament were separately measured using the HR4000 spectrometer nearby the filament. A power of $158 \mathrm{~W}$ was applied to obtain a spectrum that covers all the wavelength ranges of the optical filter used in this detection system. The spectrum at $158 \mathrm{~W}$ was well reproduced by the Plank's formula at $2550 \mathrm{~K}$. The count rates measured with optical filters divided by those without filters were compared with the black body radiation curve, and a set of calibration factors, which represent the efficiencies of filters and photomultipliers, were obtained. Figure 3 shows the data of lower temperature tungsten filament before (open circle) and after (solid square) applying the normalization factor together with the black body spectrum at $2160 \mathrm{~K}$ that reproduced the spectrum separately measured with the HR4000 spectrometer (dashed line). The data measured with the present detection system agreed with the black body spectrum at $2160 \mathrm{~K}$.

As an example of the obtained spectra, Fig. 4 exhibits the spectrum obtained with a $9 \mathrm{kV}$ Ar cluster beam, impinging on a silicon surface. A $\mathrm{Si}(100)$ sample was cleaned using purified water, organic solvents, sulfuric acid/hydrogen peroxide mixture, and hydrofluoric acid prior to the introduction to the vacuum chamber. The beam spot on the sample had an approximately $9 \mathrm{~mm}$ diameter circular shape. The naturally oxidized layer formed after the cleaning was expected to be removed by the high sputtering yield of Ar cluster beam. ${ }^{18,19}$ It is possible to see a broad peak whose maximum corresponds to the wavelength of neutral Ar spectrum obtained from NIST database. ${ }^{20}$ The intensities listed in the NIST database were added within the wavelength ranges of the optical filters. The vertical scale was normalized to the data at $429 \mathrm{~nm}$. A preliminary calculation of the temperature achieved by clusters during the collision process has been performed using the ratio between the values of the emission at $429 \mathrm{~nm}$ obtained at $9 \mathrm{kV}$ and $6 \mathrm{kV}$ acceleration voltages. Supposing the collision process is approximately adiabatic, and thus the temperature at $9 \mathrm{kV}$ is about $3 / 2$ of that at $6 \mathrm{kV}$, the temperature at $9 \mathrm{kV}$ should be larger than $10000 \mathrm{~K}$. This information confirms that collision process produces high temperatures in the collision area, as foreseen by molecular dynamic simulations.

\section{SUMMARY}

A spectrometer using photomultipliers and band-pass optical filters was developed to detect the photons emitted during the collision of cluster ions against solid surfaces. This spectrometer enables the experimental research on the collision process of cluster ions, which may contribute to the better understanding of the behavior of nanosystems. It may permit to check the previsions of the MD simulations present in literature. It may permit to discriminate between the black body radiation due to the high temperature present during collision and the emission due to the capture and consequent emission of electrons by the target material. Furthermore, this spectrometer has a possibility of application in the on-line monitoring of the surface properties during the processing using cluster ion beams.

\section{ACKNOWLEDGMENTS}

The authors thank Professor Y. Gotoh and Mr. H. Tsuji of Kyoto University for their help in the MPS-2000 and HR4000 spectrometers.

${ }^{1}$ P. D. Townsend, P. J. Chandler, and L. Zhang, Optical Effects of Ion Implantation (Cambridge University Press, Cambridge, 1994), Chap. 4.

${ }^{2}$ K. G. Malmqvist, M. Elfman, G. Remond, and C. Yang, Nucl. Instrum. Methods Phys. Res., Sect. B 109/110, 227 (1996).

${ }^{3}$ R. J. Brooks, D. E. Hole, and P. D. Townsend, Nucl. Instrum. Methods Phys. Res., Sect. B 190, 136 (2002).

${ }^{4}$ I. Yamada, Nucl. Instrum. Methods Phys. Res., Sect. B 148, 1 (1999).

${ }^{5}$ G. H. Takaoka, H. Ryuto, and M. Takeuchi, J. Mater. Res. 27, 806 (2012).

${ }^{6}$ C. L. Cleveland and U. Landman, Science 257, 355 (1992).

${ }^{7}$ H.-P. Chen and U. Landman, Science 260, 1304 (1993).

${ }^{8}$ H. Ryuto, F. Musumeci, A. Sakata, M. Takeuchi, and G. H. Takaoka, Rev. Sci. Instrum. 85, 02 C303 (2014).

${ }^{9}$ O. F. Hagena and W. Obert, J. Chem. Phys. 56, 1793 (1972).

${ }^{10}$ H. Ryuto, R. Araki, T. Yakushiji, and G. H. Takaoka, Trans. Mater. Res. Soc. Jpn. 35, 781 (2010).

${ }^{11}$ H. Ryuto, G. Ichihashi, M. Takeuchi, and G. H. Takaoka, Nucl. Instrum. Methods Phys. Res., Sect. B 315, 231 (2013).

${ }^{12}$ O. F. Hagena and K. Varma, Rev. Sci. Instrum. 39, 47 (1968).

${ }^{13}$ S. Schütte and U. Buck, Int. J. Mass Spectrom. 220, 183 (2002).

${ }^{14}$ C. Bobbert, S. Schütte, C. Steinback, and U. Buck, Eur. Phys. J. D 19, 183 (2002).

${ }^{15}$ R7400p, R7400p-01, Hamamatsu Photonics, Hamamatsu, Japan.

${ }^{16}$ FILTROP AG, Balzers, Lichtenstein.

${ }^{17}$ LumiNova, Nemoto Lumi-Materials, Tokyo, Japan.

${ }^{18}$ J. Matsuo, N. Toyoda, M. Akizuki, and I. Yamada, Nucl. Instrum. Methods Phys. Res., Sect. B 121, 459 (1997).

${ }^{19}$ T. Seki, T. Murase, and J. Matsuo, Nucl. Instrum. Methods Phys. Res., Sect. B 242, 179 (2006).

${ }^{20}$ NIST Atomic Spectroscopy Databases, see http://www.nist.gov/pml/data/ atomspec.cfm. 